\section{Les négociations TarMed}

Groupe de travail TarMed de la FMH

Hhb. Les négociations concernant le TarMed (pour ce qui est de l'organisation et de la composition, nous vous renvoyons à l'article sur ce sujet) n'ont plus grand-chose à voir avec les négociations d'autrefois.

La base des négociations est une banque de données relationnelle comprenant une nomenclature des prestations médicales, des données empiriques liées à l'économie d'entreprise, des variables de pilotage et des algorithmes de calcul; ces derniers servent à relier les données et les variables selon un nombre de possibilités potentiellement illimité. Bien évidemment, tout cela n'est réalisable que grâce à l'électronique et à des moyens informatiques modernes.

Cela étant, les négociations consistent pour l'essentiel à déterminer les données et les variables de pilotage devant servir aux calculs de tarification. Or, cette démarche se fait non pas pour chaque prestation isolément, mais pour l'ensemble de la structure tarifaire ou pour de grandes parties de celle-ci. Lors du processus visant à fixer les données, notamment de celles servant au pilotage, on peut procéder à des estimations de leur effet sur de grands volumes de facturation, mais jamais sur une prestation particulière.

Après une nouvelle série de factures, il s'agit en principe de vérifier les effets sur chacune des prestations. Si ceux-ci sont indésirables, voire inacceptables, les décisions et les variables de pilotage sont à leur tour vérifiées et, le cas échéant, modifiées; puis vient une autre série de factures, etc., etc.

A la demande d'une grande partie du corps médical, la version 1.0 a été publiée sans vérification approfondie. Il est certain que les sociétés de discipline médicale intéressées y auront trouvé certaines erreurs, des illogismes et d'autres choses encore; c'est d'ailleurs pourquoi nous avons émis le vœu que de telles constatations soient communiquées immédiatement au service tarifaire. Ce dernier inclura ces remarques ainsi que les résultats de la vérification interne dans une banque de données qui servira de base pour les travaux ultérieurs.

En bref, il ressort de ces explications que:

1. l'idée que chaque prestation est négociée, modifiée, etc., isolément est fausse;

2. toute information sur les changements intervenant pour des prestations isolées ou des groupes de prestations particuliers ne pourra être donnée qu'après un certain nombre de factures;

3. le reproche que l'on aurait intentionnellement procédé à des changements "dans le dos des intéressés" est sans objet, car il repose sur de fausses prémisses. Ces visions récurrentes de boîtes noires, de vauriens préparant des mauvais coups dans une arrière-salle enfumée et de saboteurs trafiquant les banques de données pendant la nuit, tout cela n'est que pure fiction. Peut-être y a-t-il eu de telles manigances à l'époque des négociations sur les tarifs de bazar, où tout fonctionnait selon le principe du "do ut des», autrement dit du «donnant, donnant». Avec le TarMed, les négociations de ce genre, où l'on échangeait une pomme contre une poire, ont été reléguées aux oubliettes;

4. les personnes - car c'est bien de personnes qu'il s'agit et non de robots, comme le présumait récemment l'ancien président de la Bernoise - qui travaillent à ces systèmes sont souvent étonnées, voire perplexes, et parfois même désemparées un bref instant. Ce ne sont pourtant pas des idiots incompétents; on pourrait les comparer à des ingénieurs travaillant à la mise au point d'un prototype d'avion, la seule véritable différence étant que de tels ingénieurs n'auraient pas à se débattre dans l'arène devant des milliers de clients potentiels, de responsables politiques et de représentants des médias dont la compétence présumée en ingénierie est légitimée par le fait qu'ils ont tous pris un avion au moins une fois dans leur vie. 